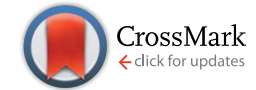

Cite this: RSC Adv., 2014, 4, 56156

\title{
45S5 bioactive glass-based scaffolds coated with cellulose nanowhiskers for bone tissue engineering
}

\author{
Wei Li, ${ }^{a}$ Nere Garmendia, ${ }^{b}$ Uxua Pérez de Larraya, ${ }^{\text {b }}$ Yaping Ding, ${ }^{c}$ Rainer Detsch, ${ }^{a}$ \\ Alina Grünewald, ${ }^{a}$ Judith A. Roether, ${ }^{c}$ Dirk W. Schubert ${ }^{c}$ and Aldo R. Boccaccini ${ }^{\star a}$
}

\begin{abstract}
Highly porous 4555 bioactive glass-based scaffolds prepared by foam replication method were coated with cellulose nanowhiskers by dip coating method. The obtained cellulose nanowhisker-coated scaffolds retained the high porosity and interconnected pore structure. The cellulose coating improved the mechanical properties of the scaffolds and did not hinder their bioactivity in simulated body fluid. In vitro biocompatibility assessment was carried out by qualitative evaluation of the morphology of osteoblastlike cells (MG-63) seeded onto the scaffolds. The cells were shown to attach and spread on both uncoated scaffolds and cellulose nanowhisker-coated scaffolds, thus cellulose nanowhisker coating seems to have no negative influence on the behavior of MG-63 cells. The obtained bioactive and biocompatible composite scaffolds represent promising candidates for bone tissue engineering applications.
\end{abstract}

Received 28th July 2014

Accepted 10th October 2014

DOI: $10.1039 / c 4 r a 07740 \mathrm{~g}$

www.rsc.org/advances

a recent study, for the first time also, microfibrillated cellulose,

\section{Introduction}

With an increasing aging population, there is a high demand for bone grafts for repairing bone defects caused by trauma, tumor or bone related diseases. ${ }^{1}$ The gold standard for bone defect repairing is autologous bone grafting due to its high immunocompatibility. However, autologous bone grafting is bound by several constraints, such as limited amount of tissue that can be harvested, requirement of a secondary surgery and risk of infection. ${ }^{1,2}$ Thus, a large number of bone substitutes made of engineered materials are under investigation in the context of tissue engineering. Such substitutes, called scaffolds, exhibit 3D high porosity and suitable surface properties and degradation behavior. ${ }^{1,3}$

45S5 bioactive glass (BG) is a promising material for fabricating bone tissue engineering scaffolds, since its excellent bioactivity, biocompatibility, osteogenic and angiogenic effects $^{4-7}$ meet relevant requirements for ideal scaffolds. ${ }^{\mathbf{1 , 8}, \mathbf{9}}$ Highly porous $45 \mathrm{~S} 5$ BG-based scaffolds fabricated by foam replication method are strong enough for manual handling, however the obtained mechanical properties (e.g. compressive strength) are still not enough for bone tissue engineering application in load-bearing conditions. ${ }^{10}$ To this end, different polymers are being used to coat 45S5 BG-based scaffolds. ${ }^{11-15}$ In

${ }^{a}$ Institute of Biomaterials, Department of Materials Science and Engineering, University of Erlangen-Nuremberg, Cauerstrasse 6, 91058 Erlangen, Germany. E-mail: aldo.boccaccini@ww.uni-erlangen.de; Fax: +49 913185 28602; Tel: +49 91318528601

${ }^{b}$ Cemitec, Polígono Mocholi, Plaza Cein 4, 31110 Noain, Navarra, Spain

'Institute of Polymer Materials, Department of Materials Science and Engineering, University of Erlangen-Nuremberg, Martensstrasse 7, 91058 Erlangen, Germany mixed with polyvinyl alcohol, was used as a composite coating on 45S5 BG-based scaffolds. ${ }^{14}$ Moreover, the drug release function of the applied polymer coatings has been investigated in some studies. ${ }^{12,16,17}$ The results showed that the polymer coatings, including both synthetic and natural polymers, can improve the compressive strength and work of fracture of $45 \mathrm{~S} 5$ BG-based scaffolds. In addition, a sustained drug release profile can be achieved by the presence of polymer coatings, i.e., when the drug is incorporated in the biodegradable polymer.

Generally, polymers used to coat scaffolds, whether synthetic or natural, are completely dissolved in solvents, e.g. organic solvents (chloroform or dimethyl carbonate) or water, to prepare the coatings, which are usually obtained by dip coating the scaffolds into the polymer solution. ${ }^{12,13,15,18}$ Through this dissolution-precipitation coating procedure, the obtained polymer layer, possessing either compact or porous network structure, exhibits a surface topography in the microscale..$^{12,13,19}$ Given that the surface topography of biomaterials, in micrometer and nanometer scale, affects the attachment, proliferation and differentiation of cells, ${ }^{20-22}$ it is of high interest to incorporate nanosize features on the 3D surfaces of the scaffolds. ${ }^{23}$

One interesting approach to achieve this goal is to develop a polymer coating with nanoscale topography covering the surface of scaffolds thus mimicking the nanoscale structure of the natural extracellular matrix (ECM). Only a limited number of reports is available in the literature on the incorporation of nanoscale fillers into polymer coatings for bioactive glass and bioceramic scaffolds. ${ }^{14,24,25}$

In the present investigation, cellulose nanowhiskers alone, rather than microfibrillated cellulose related composite, ${ }^{14}$ were 
chosen for the first time in order to obtain a nanostructured polymer coating. Cellulose nanowhiskers, also called cellulose nanocrystals, are isolated from natural cellulosic materials by acid hydrolysis. They are the crystalline part of cellulose and have a rod like shape. ${ }^{26}$ For numerous applications, including the biomedical field, cellulose nanowhiskers are gaining a tremendous level of attention due to their unique physical and chemical properties. ${ }^{26-31}$ In the context of biomedical applications, these nanomaterials are considered promising candidates thanks to their biocompatibility, functionality, low density, high specific strength and modulus..$^{26,27,32}$ Until now, only a relatively limited number of in vitro studies have been reported about the interactions between cellulose nanowhiskers or their composites and living cells. Cells investigated in previous studies were for example endothelial cells, ligament cells and fibroblasts, ${ }^{33-39}$ in addition to human adult adipose derived mesenchymal stem cells which were cultured on electrospun fibers reinforced with cellulose nanocrystals. ${ }^{40}$

To the best of our knowledge, the combination of bioactive glass scaffolds and nanostructured coating incorporating cellulose nanowhiskers has not been investigated before. In particular, the in vitro biocompatibility of cellulose nanowhiskers reinforced 45S5 BG-based composite has not been reported. Hence, the aim of this study was to develop the technology to fabricate highly porous 45S5 BG-based scaffolds coated with cellulose nanowhiskers, and to characterize the new scaffolds with focus on investigating the possible influence on bioactivity and biocompatibility that the cellulose nanowhiskers can have on the standard 45S5 BG-based scaffolds.

\section{Materials and methods}

\subsection{Materials}

Microcrystalline cellulose used for preparing cellulose nanowhiskers was purchased from Sigma-Aldrich (St. Louis, MO, USA). Commercially available melt-derived 45S5 BG powder of particle size $\sim 5 \mu \mathrm{m}$ was used for fabricating scaffolds. Polyurethane (PU) foam (45 pores per inch) was obtained from Eurofoam (Troisdorf, Germany). Polyvinyl alcohol (PVA, completely hydrolyzed, $M_{\mathrm{W}}=30000 \mathrm{~g} \mathrm{~mol}^{-1}$ ) was obtained from Merck (Darmstadt, Germany). All the other chemicals for simulated body fluid (SBF) and phosphate buffered saline (PBS) preparation were purchased from Sigma-Aldrich (St. Louis, MO, USA).

\subsection{Fabrication of 4555 BG-based scaffolds}

The scaffolds were fabricated by the foam replication method, as described in detail elsewhere. ${ }^{10,12}$ Briefly, PVA was dissolved in deionized water at a concentration of $6 \% \mathrm{w} / \mathrm{v}$ at $80{ }^{\circ} \mathrm{C}$, and then 45S5 BG powder was added to the PVA solution up to a concentration of $50 \mathrm{wt} \%$ under vigorous stirring to obtain homogeneous slurry. PU foams with dimensions of $15 \mathrm{~mm} \times 15$ $\mathrm{mm} \times 12 \mathrm{~mm}$ were immersed in the slurry and rotated to ensure homogeneous slurry infiltration. Each procedure was carried out using a magnetic stirrer. The scaffolds were then extracted from the slurry, and the extra slurry was completely squeezed out. The samples were dried at room temperature for $24 \mathrm{~h}$ and then the procedure described above was repeated again. The samples were then heated at $400{ }^{\circ} \mathrm{C}$ for $1 \mathrm{~h}$ in air to decompose the $\mathrm{PU}$ foam, and then at $1100{ }^{\circ} \mathrm{C}$ for $2 \mathrm{~h}$ to densify the struts. The heating and cooling rates used were $2{ }^{\circ} \mathrm{C} \min ^{-1}$ and $5{ }^{\circ} \mathrm{C} \min ^{-1}$, respectively.

\subsection{Fabrication of cellulose nanowhiskers}

Cellulose nanowhiskers were prepared from microcrystalline cellulose by acid hydrolysis. The reaction was performed using $63.5 \% \mathrm{w} / \mathrm{w}$ sulfuric acid $\left(10 \mathrm{~mL} \mathrm{H}_{2} \mathrm{SO}_{4} / 1 \mathrm{~g}\right.$ MCC) at $44{ }^{\circ} \mathrm{C}$ for $2 \mathrm{~h}$ under mechanical stirring and stopped by diluting with 10 -fold cold $\left(4^{\circ} \mathrm{C}\right)$ water. The obtained suspension was concentrated and subsequently washed with deionized water by repeated centrifuged cycles at $9000 \mathrm{rpm}$ for $10 \mathrm{~min}$ until the supernatant became turbid. Then, the suspension was dialyzed in water until a constant $\mathrm{pH}$ was reached and finally ultrasonicated for 10 minutes. The final suspension concentration was at $0.42 \% \mathrm{w} / \mathrm{v}$.

\subsection{Polymer coating procedure}

Prepared 45S5 BG-based scaffolds were coated with cellulose nanowhiskers by a simple dip coating procedure. Scaffolds with dimensions $10 \mathrm{~mm} \times 8 \mathrm{~mm} \times 8 \mathrm{~mm}$ were dipped in $100 \mathrm{~mL}$ of cellulose nanowhiskers aqueous suspension $(0.42 \% \mathrm{w} / \mathrm{v})$ for 1 minute and then dried at room temperature.

\subsection{Characterization methods}

2.5.1. Porosity. The porosities of scaffolds before $\left(p_{1}\right)$ and after $\left(p_{2}\right)$ coating were calculated by eqn (1) and (2), respectively:

$$
\begin{gathered}
p_{1}=1-M_{1} /\left(\rho_{\mathrm{BG}} V_{1}\right) \\
p_{2}=1-\left(M_{1} / \rho_{\mathrm{BG}}+\left(M_{2}-M_{1}\right) / \rho_{\text {cellulose }}\right) / V_{2}
\end{gathered}
$$

where $M_{1}$ and $M_{2}$ are the mass of the scaffolds before and after coating with cellulose, respectively; $V_{1}$ and $V_{2}$ are the volume (calculated from the dimensions) of the scaffolds before and after coating with cellulose, respectively; $\rho_{\mathrm{BG}}\left(=2.7 \mathrm{~g} \mathrm{~cm}^{-3}\right)$ is the density of $45 \mathrm{~S} 5 \mathrm{BG}$ by assuming that crystallization would not significantly change this value, ${ }^{\mathbf{4 1 , 4 2}}$ and $\rho_{\text {cellulose }}(=1.6 \mathrm{~g}$ $\mathrm{cm}^{-3}$ ) is the density of cellulose. ${ }^{43}$

2.5.2. Surface morphology. The microstructure of the scaffolds was characterized using scanning electron microscopy (SEM) (LEO 435 VP, Cambridge, UK and Ultra Plus, Zeiss, Germany). In order to better observe the morphology of cellulose nanowhiskers in the nanoscale, a drop of diluted cellulose nanowhisker solution $(0.05 \% \mathrm{w} / \mathrm{v})$ was placed on a coppercarbon grid. This sample was dried at room temperature and then directly observed by SEM. Other samples were sputtered with gold (Edwards, S150B, Sussex, UK) before observation.

2.5.3. Contact angle measurement. DSA30 contact angle measuring instrument (Kruess, Germany) was used to determine the static contact angle on disk samples. The 45S5 BG disks were produced by uniaxial pressing of the 45S5 BG powder in a cylindrical die with a diameter of $15 \mathrm{~mm}$, followed by sintering using the same heat treatment used for the scaffolds. The 
sintered 45S5 BG disks were coated with the same cellulose nanowhisker solution using the same procedure described for coating the scaffolds. By comparison, cellulose nanowhisker films were also prepared by casting the cellulose nanowhisker solution into petri dishes. Water with a volume of $3 \mu \mathrm{L}$ was added on the samples by a motor-driven syringe at room temperature. The results were reported by averaging the results of five measurements.

2.5.4. Mechanical testing. The mechanical properties of scaffolds $(10 \mathrm{~mm} \times 8 \mathrm{~mm} \times 8 \mathrm{~mm})$ before and after coating with cellulose nanowhiskers were measured using a Zwick/Roell Z050 mechanical tester operated at a crosshead speed of $0.5 \mathrm{~mm} \mathrm{~min}{ }^{-1}$. The load cell has a loading capacity of $50 \mathrm{~N}$. During compressive strength test, the load was applied on the $8 \mathrm{~mm} \times 8 \mathrm{~mm}$ plane of the scaffolds until the compressive strain reached $70 \%$. The compressive strength was determined from the maximum load of the obtained stress-strain curve before the compaction of the scaffolds. An estimation of the work of fracture $\left(W_{\mathrm{ab}}\right)$ for the uncoated and coated scaffolds, which is related to the energy necessary to deform a sample to a certain strain, was obtained from the area under the corresponding load-displacement curve up to $70 \%$ strain. At least five samples were tested for each condition. Average values and standard deviations were determined. In addition, in order to more closely imitate the actual in vivo environment in the intended application in bone tissue engineering, the mechanical properties of the scaffolds before and after immersion in SBF were tested in wet condition, i.e., the scaffolds were soaked in PBS at $37^{\circ} \mathrm{C}$ before compressive strength test.

\subsection{In vitro bioactivity assessment}

The in vitro bioactivity test was carried out using the standard procedure described by Kokubo et al. ${ }^{44}$ The scaffolds $(10 \mathrm{~mm} \times$ $8 \mathrm{~mm} \times 8 \mathrm{~mm}$ ) were immersed in $50 \mathrm{~mL}$ SBF and kept in a shaking incubator at $37^{\circ} \mathrm{C}$ and $90 \mathrm{rpm}$. Samples were collected after 1, 3, 7 and 14 days of immersion, during which the SBF was replaced twice a week. Once removed from the incubator, the samples were rinsed with deionized water and left to dry at room temperature in a desiccator for further FTIR (Nicolet 6700, Thermo Scientific, USA) and SEM examination. For FTIR, the scaffolds were ground, mixed with $\mathrm{KBr}$ (spectroscopy grade, Merck, Germany) and pressed into pellets. The pellets were made by mixing $1 \mathrm{mg}$ of sample and $200 \mathrm{mg}$ of $\mathrm{KBr}$. Spectra were recorded in absorbance mode between 2000 and $400 \mathrm{~cm}^{-1}$ with a resolution of $4 \mathrm{~cm}^{-1}$.

\subsection{In vitro biocompatibility assessment}

2.7.1. Preparation of cells and scaffolds. Before cell seeding, scaffolds were cleaned by soaking in Extran (Merck, Germany) and SDS (sodium dodecyl sulphate, Sigma-Aldrich, Germany) solutions. Afterwards, uncoated scaffolds were sterilized at $160{ }^{\circ} \mathrm{C}$ for $7 \mathrm{~h}$ in a furnace (Nabertherm, Germany). Cellulose nanowhiskers coated scaffolds were sterilized by immersion in ethanol for $1 \mathrm{~h}$, then washed with sterile PBS for 3 times and left to dry in the sterile bench. Four replicates of each type of sample for several experiments, which were repeated three times, were pre-incubated with cell culture medium for 7 days.

2.7.2. Cell culture. To evaluate the cell behavior of osteoblast-like cells on different scaffolds, 1 million MG-63 cells (Sigma-Aldrich, Germany) in $1 \mathrm{~mL}$ cell culture medium were seeded on each scaffold, and cells were cultivated for 2 and 3 weeks with change of culture medium every 2-3 days. In general, MG-63 cells were cultured at $37{ }^{\circ} \mathrm{C}$ in a humidified atmosphere of $95 \%$ air and 5\% $\mathrm{CO}_{2}$, in DMEM (Dulbecco's modified Eagle's medium, Gibco, Germany) containing 10 vol\% fetal bovine serum (FBS, Sigma-Aldrich, Germany) and 1 vol\% penicillin/streptomycin (Gibco, Germany). Cells were grown to confluence in $75 \mathrm{~cm}^{2}$ culture flasks (Nunc, Denmark), and afterwards harvested using Trypsin/EDTA (Gibco, Germany) and counted by a hemocytometer (Roth, Germany).

After 2 and 3 weeks of MG-63 cell incubation into the scaffolds, cell distribution, attachment and cell morphology were qualitatively determined. To visualize the adherent grown cells on the scaffold samples, Vybrant ${ }^{\mathrm{TM}}$ cell-labelling solution (Molecular Probes, The Netherlands) was used. After several incubation times, cell culture medium was removed and staining solution ( $5 \mu \mathrm{L}$ dye labelling solution to $1 \mathrm{~mL}$ of growth medium) was added and incubated for 15 min. Afterwards the solution was removed, the samples were washed with PBS (Gibco) and cells on the surfaces were fixed by 3.7 vol\% paraformaldehyde. Samples were washed again and left in PBS for microscopic viewing with a confocal scanning laser microscope (CSLM, Leica TCS SP5 II, Germany). The CLSM images were taken from the outside surface of the scaffolds. For cell morphology characterization, cells on scaffolds were fixed in 3 vol\% paraformaldehyde, 3 vol\% glutaraldehyde (Sigma-Aldrich, Germany) and 0.2 M sodiumcacodylate (Sigma-Aldrich, Germany). After dehydration through incubation with a series of graded ethanol series $(30,50,70,80,90,95$ and 100 vol\%), the samples were critical point dried with $\mathrm{CO}_{2}$ (EM CPD300, Leica, Germany) and sputtered with gold. The cell morphology of the inner part of scaffolds was analyzed by SEM.

\section{Results and discussion}

\subsection{Microstructure characterization}

Fig. 1(a) and (b) show the highly interconnected pore structure and the surface morphology of the strut of uncoated scaffolds, respectively. After coating, the open pore structure was well maintained, as confirmed by Fig. 1(c). The polymer coating only blocked few pores. Most of the struts were covered by a thin layer of cellulose, although it was observed that struts were only partially coated (Fig. 1(d)). Coating thickness values of maximum $\sim 2 \mu \mathrm{m}$ were estimated from SEM observations. There were no attempts at correcting this result, because from the biological point of view, this type of coating morphology is favorable to maintain the intrinsic bioactivity of the scaffolds, since the uncoated areas of struts will be directly exposed to biological fluids enabling the typical surface reactions of the bioactive glass-ceramic to take place. ${ }^{12,15}$ Fig. $1(\mathrm{e})$ indicates that the obtained cellulose coating is quite dense at least in the microscale, and the cellulose coating was qualitatively 

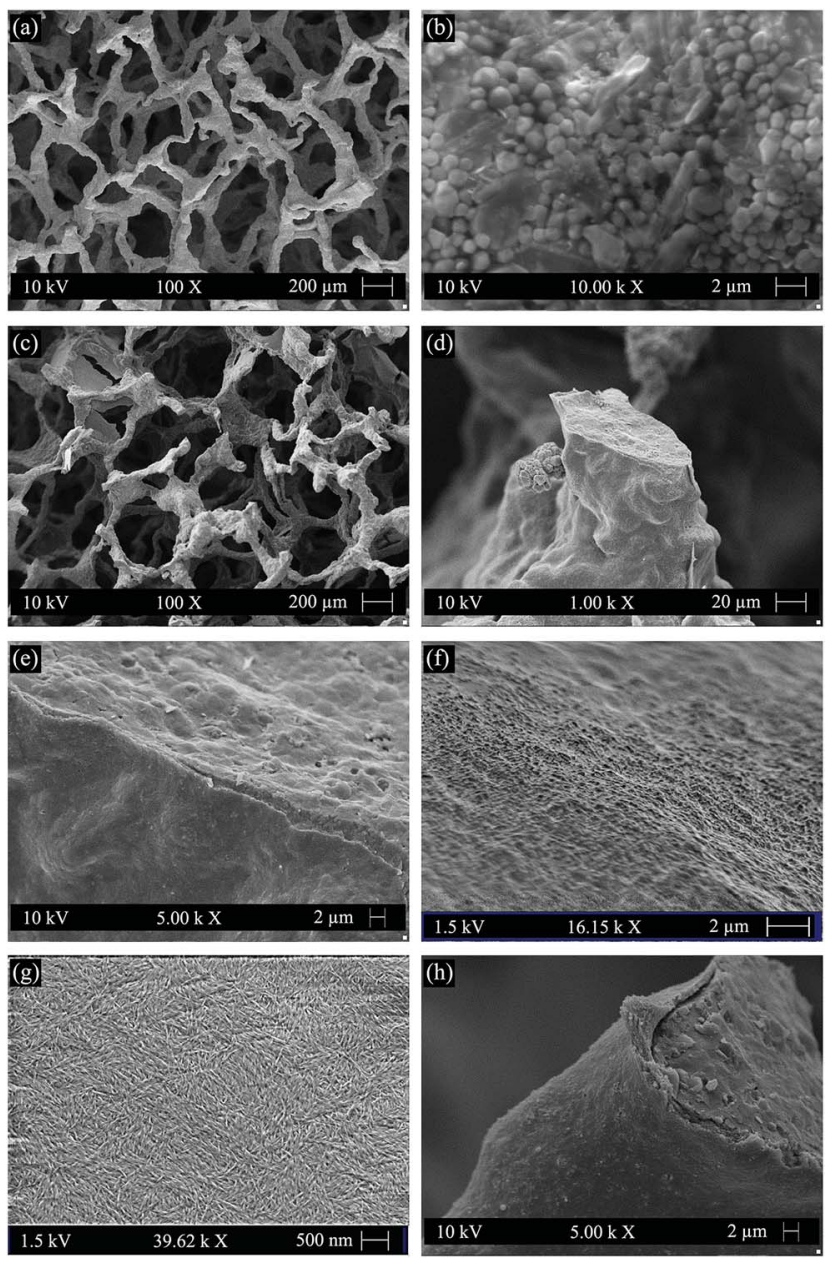

Fig. 1 SEM micrographs of (a) and (b) uncoated scaffolds, (c)-(f) cellulose nanowhiskers coated scaffolds, (g) cellulose nanowhiskers at different magnifications and (h) cellulose nanowhiskers coated scaffolds after immersion in SBF for 1 day.

confirmed to adhere well to the surface of scaffold struts. This qualitative good adhesion of cellulose to 45S5 BG glass-ceramic is likely due to the hydrophilic character of these two materials. ${ }^{12,27}$ Fig. 1(f) shows the surface morphology of the cellulose coated strut at higher magnification indicating that it is relatively smoother than the uncoated strut (Fig. 1(b)). Fig. 1(g) shows the morphology of cellulose nanowhiskers in nanoscale, in which individual cellulose nanowhiskers are visible. Since these cellulose nanowhiskers are expected to serve as a coating on the struts of the scaffolds when they are immersed in body fluid environment, the adhesion of the cellulose nanowhiskers on the struts of the scaffolds was further checked after immersion in SBF. Fig. 1(h) shows the cross section of a scaffold strut after immersion in SBF for 1 day. The cellulose coating appears well adhered to the strut, and no coating peeling off during cutting of the scaffolds occurred which qualitatively indicates that the cellulose coating was stable in the simulated body fluid environment.

The porosity of scaffolds before and after coating was calculated to be $95 \%$ and $93 \%$, respectively, which indicates that the cellulose coating only slightly reduced porosity. The pore size of cellulose coated scaffolds was in the range of 200 to $550 \mu \mathrm{m}$ as assessed by SEM images. High porosity and interconnected pore structure are favorable for osteogenesis and vascularization throughout the entire $3 \mathrm{D}$ scaffold. In addition, studies have suggested that scaffolds with pore sizes in the above mentioned range exhibit enhanced osteogenesis than those scaffolds with smaller pore sizes (such as $<200 \mu \mathrm{m}$ ). ${ }^{1,45}$

\subsection{Surface hydrophilicity measurement}

Water contact angle was measured to evaluate the surface hydrophilicity of the samples in the present study. Table 1 shows the contact angles of the uncoated 45S5 BG disk, cellulose nanowhiskers coated 45S5 BG disk and cellulose nanowhisker film. The uncoated 45S5 BG exhibited a typical low contact angle due to the intrinsic hydrophilic surface of $45 \mathrm{~S} 5$ BG. The contact angle of $45 \mathrm{~S} 5$ BG disk was increased in the presence of cellulose nanowhisker coating. However, it was still significantly lower than that of the cellulose nanowhisker film, indicating that the $45 \mathrm{~S} 5 \mathrm{BG}$ disk was not covered by a continuous layer of cellulose nanowhisker film. In other words, the 45S5 BG disk was only partly coated by the cellulose nanowhiskers, which is also in agreement with the SEM micrograph of the struts of coated scaffolds (Fig. 1(d)). It is anticipated that the relatively hydrophilic surface of cellulose nanowhiskers coated scaffolds will not hinder their bioactivity and interactions with cells.

\subsection{Mechanical properties}

Typical compressive stress-strain curves of uncoated and cellulose nanowhiskers coated scaffolds are shown in Fig. 2 . The average compressive strengths of uncoated and coated scaffolds were determined to be $0.02 \pm 0.01 \mathrm{MPa}$ and $0.06 \pm$ $0.01 \mathrm{MPa}$, respectively. The area under the load-displacement curve, as an indication of the work of fracture for the uncoated and coated scaffolds, was calculated to be $3.1 \pm 0.3 \mathrm{~N} \mathrm{~mm}$ and $13.5 \pm 1.8 \mathrm{~N} \mathrm{~mm}$, respectively. Obviously, the cellulose coating did improve the compressive strength of the scaffolds and made the scaffolds much tougher. Since the porosity of the scaffolds only slightly reduced from $95 \%$ to $93 \%$ after coating, it is apparent that it is the presence of the cellulose coating having such notable effect on the mechanical properties of scaffolds.

A wet environment is closer to real physiological (in vivo) conditions. Therefore, the compressive strength of the uncoated and cellulose nanowhisker coated scaffolds was also tested in wet condition and the results are shown in Fig. 3. For uncoated scaffolds, the compressive strength slightly increased in wet condition, and then slightly decreased after immersion in SBF for 7 days. On the other hand the compressive strength of the cellulose nanowhisker coated scaffolds slightly decreased in wet condition, and further decreased after immersion in SBF for 7 days.

In terms of increased degree of compressive strength and work of fracture, the strengthening and toughening effects of cellulose nanowhiskers on scaffolds in the present study are not as significant as when PHBV, chitosan-polycaprolactone or 
Table 1 Contact angle values of uncoated 45S5 BG disk, cellulose nanowhiskers coated 45S5 BG disk and cellulose nanowhisker film

\begin{tabular}{llll}
\hline Sample & Uncoated 45S5 BG disk & Cellulose nanowhiskers coated 45S5 BG disk & Cellulose nanowhisker film \\
\hline Contact angle $^{\circ}$ & $14 \pm 4$ & $27 \pm 2$ & $62 \pm 2$
\end{tabular}

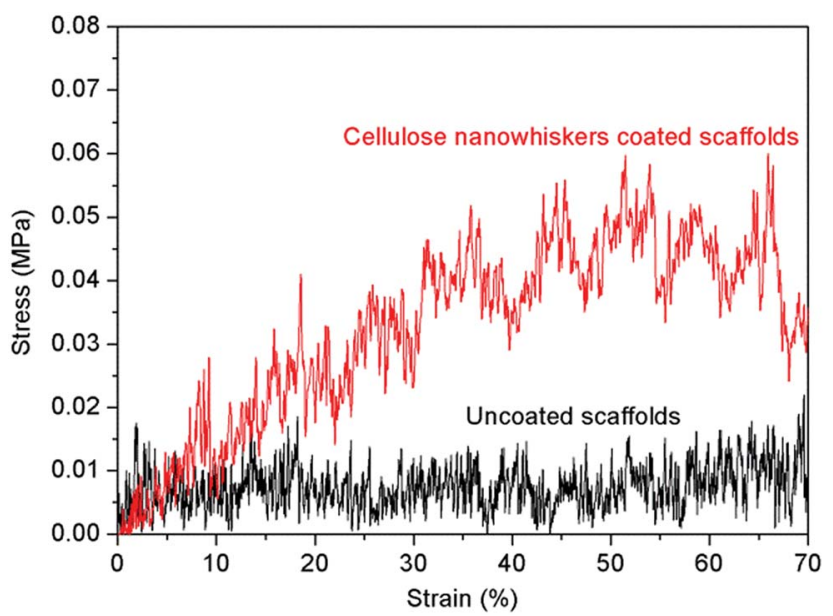

Fig. 2 Typical compressive stress-strain curves of uncoated and cellulose nanowhiskers coated scaffolds.

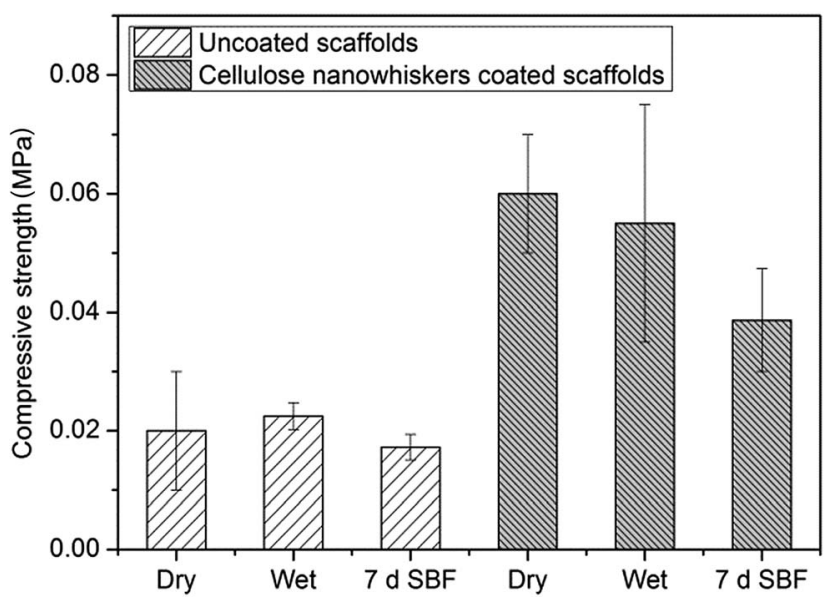

Fig. 3 Compressive strength of uncoated and cellulose nanowhisker coated scaffolds tested in dry condition, wet condition and after immersion in SBF for 7 days.

PDLLA are used as coating, ${ }^{12,18,46}$ however the effect of the coating is better than that of $\mathrm{P}(3 \mathrm{HB})$ and $\mathrm{PHBV}$ microsphere coatings. ${ }^{47,48}$ The significant strengthening and toughening effects of polymer coating such as PHBV, PDLLA or chitosanpolycaprolactone blend on scaffolds are attributed to the activation of micron scale crack-bridging mechanisms. ${ }^{12,15,18,46,49} \mathrm{In}$ contrast, the $\mathrm{P}(3 \mathrm{HB})$ and $\mathrm{PHBV}$ microsphere coating cannot provide sufficient crack bridging effect, since the microspheres do not form a continuous polymer film which is able to infiltrate the microcracks in the struts. From the point of view of structure, the cellulose nanowhisker coating, consisting of individual nanowhiskers (see Fig. 1(f)), is similar to the $\mathrm{P}(3 \mathrm{HB})$ and PHBV microsphere coating which consists of individual microspheres. ${ }^{47,48}$ Due to the small size of cellulose nanowhiskers, the obtained coating is more dense than that obtained by using microspheres. In addition, cellulose nanowhiskers tend to form strong network through hydrogenbonding and mechanical interlocking between whiskers. ${ }^{27}$ Thus, the strengthening and toughening effects of cellulose nanowhisker coating are stronger than that of loose microsphere coating. On the other hand, the cellulose network formed through hydrogen-bonding and mechanical interlocking is generally weaker than the polymer network with chain entanglement effect of molecule level. As a consequence, cellulose nanowhisker coating does not provide as significant strengthening and toughening effects as those obtained by continuous polymer coating.

By increasing the concentration of polymer solution used for coating scaffolds or by repeating the dip coating process for several times, the coating thickness will increase and therefore uncoated areas of the scaffold struts will be gradually coated by the polymer, which could further enhance the mechanical properties of the scaffolds. ${ }^{50}$ However, as indicated in Section 3.1, the thickness of polymer coating in this study was not further increased in order to balance the two properties of interest, i.e. mechanical properties and bioactivity. Clearly, the increase of coating thickness will be unfavorable for the scaffold bioactivity since the direct contact of the bioactive glass surface with the biological environment will be reduced (or even impeded) by the presence of the coating.

\subsection{In vitro bioactivity}

Hydroxyapatite formation on the surface of cellulose nanowhiskers coated scaffolds upon immersion in SBF, as an assessment of bioactivity, was investigated by using FTIR and SEM. Typical FTIR spectra of coated scaffolds before ( 0 day) and after immersion in SBF for 1, 3, 7 and 14 days are presented in Fig. 4. The FTIR spectrum of a coated scaffold after 7 days immersion in SBF clearly shows two bands at $566 \mathrm{~cm}^{-1}$ and 603 $\mathrm{cm}^{-1}$ corresponding to the $\mathrm{P}-\mathrm{O}$ bending vibrations in crystalline HA. ${ }^{51-53}$ Furthermore, the band at $873 \mathrm{~cm}^{-1}$ and the dual broad bands at $1420-1450 \mathrm{~cm}^{-1}$ can be assigned to the stretching vibration of $\mathrm{C}-\mathrm{O}$ bond, indicating that the formed $\mathrm{HA}$ was actually carbonated hydroxyapatite (HCA). ${ }^{53,54}$ These characteristic bands are not present in the spectra of scaffolds before (0 day) and after 1 day immersion in SBF, while the FTIR bands of scaffolds after 3 days immersion seem to indicate an intermediate stage of HCA formation, suggesting that apatite-like phase has likely started to form after 3 days immersion in SBF. 


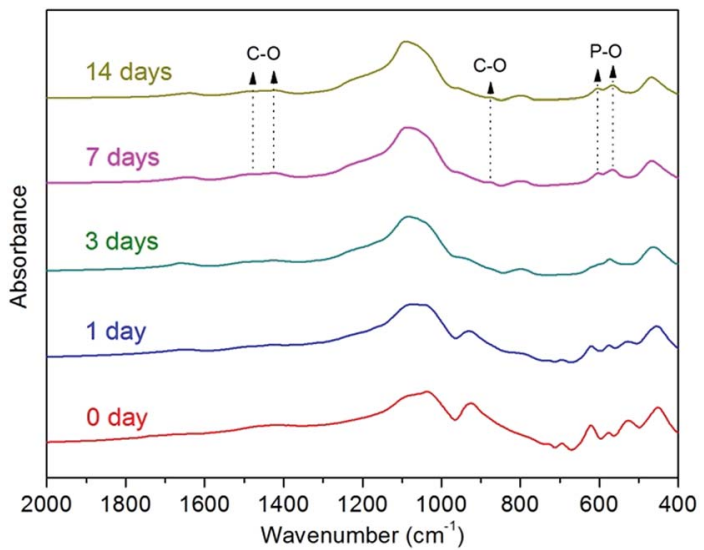

Fig. 4 FTIR spectra of coated scaffolds before ( 0 day) and after immersion in SBF for 1, 3, 7 and 14 days.

The surface morphologies of cellulose nanowhiskers coated scaffolds after immersion in SBF are presented in Fig. 5. It is observed that HCA-like crystals began to appear after 3 days immersion not only on the uncoated area of the strut but also on the cellulose coating (Fig. 5(a)). The HCA layer was quite homogeneous after 7 days immersion in SBF (Fig. 5(b)). Globular and cauliflower shape HCA crystals were clearly visible after 14 days immersion, especially at higher magnifications (Fig. 5(c) and (d)). For comparison, the surface morphologies of uncoated scaffolds after immersion in SBF are also shown in Fig. 5. At the early stage of immersion in SBF (3 days), a higher concentration of HCA-like crystals was apparent on the struts of uncoated scaffolds (Fig. 5(e)). As the immersion time increased to 7 days, the HCA layer on the struts of uncoated scaffolds (Fig. 5(f)) was similar to that on cellulose nanowhiskers coated scaffolds (Fig. 5(b)). After immersion for 14 days, the struts of uncoated scaffolds were also completely covered by the globular and cauliflower shape HCA crystals (Fig. 5(g) and (h)). Thus SEM observations indicate that the cellulose coating does not affect the surface reactivity of the scaffolds. The SEM observations agree with the FTIR. The retained bioactivity of the scaffolds after polymer coating was likely due to the existence of uncoated areas which established direct contact between the surface of the bioactive glass-ceramic strut and SBF, as reported previously for other polymer coated scaffolds. ${ }^{12}$ Furthermore, the struts were completely covered by HCA crystals after 7 and 14 days immersion in SBF, which means HCAs also grew on the cellulose coating confirming that the cellulose coating does not hinder the bioactivity of the scaffolds.

\subsection{In vitro biocompatibility}

The MG-63 osteoblast-like cells were cultured on both uncoated and cellulose nanowhiskers coated 45S5 BG-based scaffolds to assess the basic cytocompatibility of these materials and to evaluate their potential for bone tissue engineering applications. CLSM-images of uncoated and cellulose nanowhiskers coated scaffolds after incubation are shown in Fig. 6. MG-63 cells attached on both uncoated and cellulose nanowhiskers
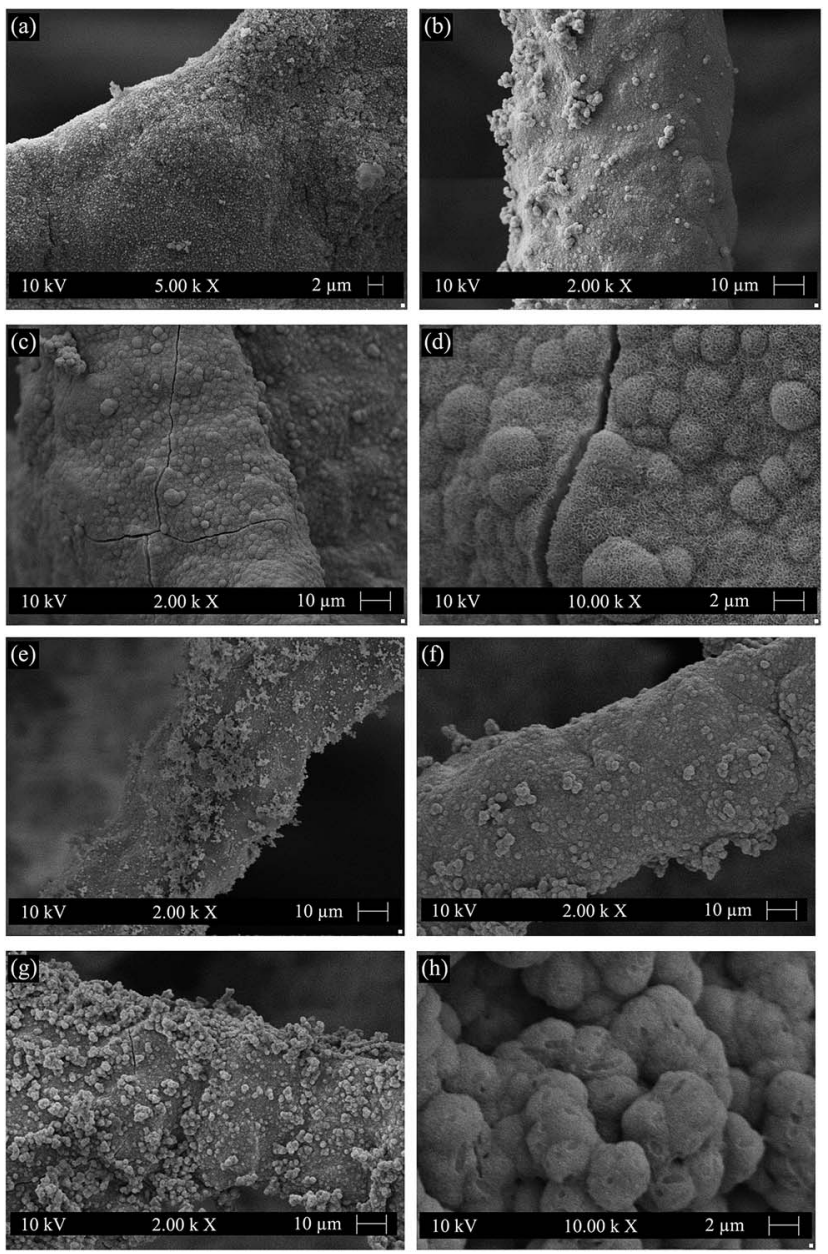

Fig. 5 SEM micrographs showing HCA formation on the surfaces of cellulose nanowhiskers coated scaffolds after immersion in SBF for (a) 3 days, (b) 7 days and (c) and (d) 14 days, and on the surfaces of uncoated scaffolds after (e) 3 days, (f) 7 days and (g) and (h) 14 days.

coated scaffolds after 2 and 3 weeks of cultivation. Specifically, the cells attached and grew on the surface of struts. As judged by visual inspection of the images, the amount of cells on both types of scaffolds after 3 weeks of cultivation seems to be higher than after 2 weeks, and the difference was particularly evident in coated scaffolds. It should be noticed that MG-63 cells can attach on the surface of both uncoated and coated scaffolds, however regarded to the organic character of cellulose nanowhiskers, MG-63 cells did not seem to spread readily on these surfaces. Thus, round shaped cells were detected on the surface of coated scaffold (Fig. 6). After 2 weeks of cultivation, there were obviously more cells on uncoated scaffolds than on coated scaffolds. However, the amount of cells on coated scaffolds considerably increased after 3 weeks of cultivation, and both scaffolds were comparable at 3 weeks. After cell cultivation for 2 and 3 weeks, the pores of both uncoated and coated scaffolds were still open. This result can be attributed to the high porosity and large pore size of the scaffolds which facilitate oxygen and nutrient supply for MG-63 cells.

Fig. 7 shows the morphology of MG-63 cells after 2 and 3 weeks of cultivation in the inner part of both uncoated and 

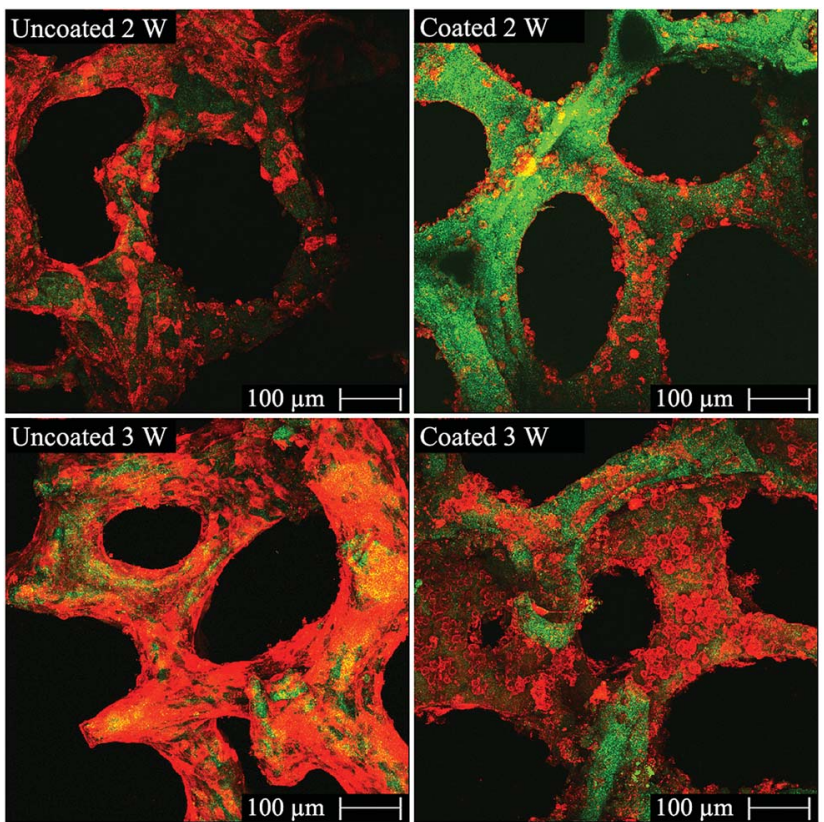

Fig. 6 CLSM-images of osteoblast-like MG- 63 cells on the surfaces of uncoated scaffolds and cellulose nanowhiskers coated scaffolds after 2 and 3 weeks of cultivation. The cells were stained red and the bioactive glass surface can be seen in green.

cellulose nanowhiskers coated scaffolds. After 2 weeks of cultivation, MG-63 cells were seen to attach and spread on the inner surface of both uncoated and cellulose nanowhiskers coated scaffolds, and these cells on both scaffold types displayed a typical osteoblastic phenotype with mainly elongated polygonal and flat structures as well as expressed filopodias in contact with the scaffold surface. Detailed images at higher magnifications (Fig. 7) show also spreaded osteoblast-like cells on the coated scaffolds. After 3 weeks of cultivation, cellular matrix production occurred on both of the cells occurred on
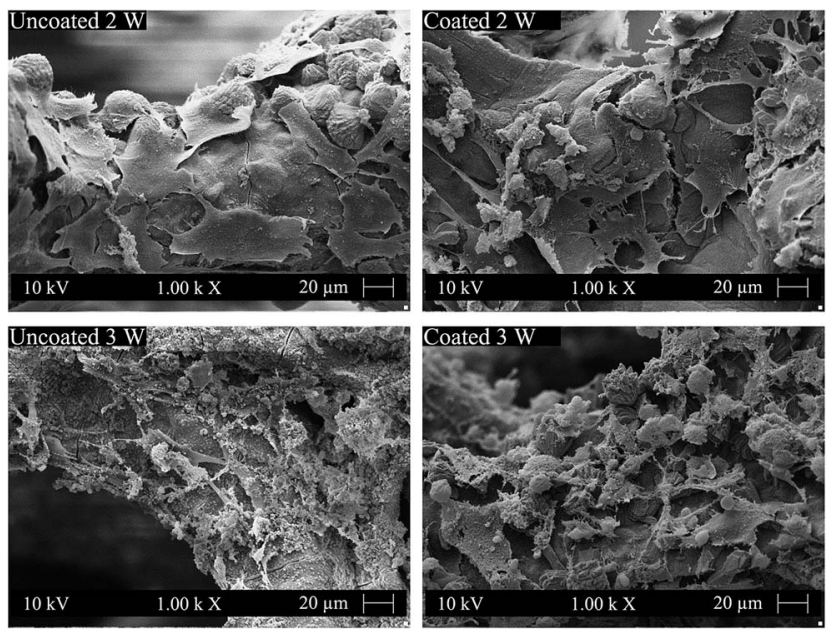

Fig. 7 SEM images of osteoblast-like MG-63 cells on the surfaces of uncoated and cellulose nanowhiskers coated scaffolds after 2 and 3 weeks of cultivation. both uncoated and cellulose nanowhiskers coated scaffolds. In contact with the culture medium, calcium phosphate also precipitated and formed small round agglomerates due to the ionic exchange between the scaffold surface and the metabolic proteins from the cell cultivation. This qualitative study has thus confirmed that MG-63 cells could well attach on both scaffolds and the cell morphology was not significantly changed by the presence of the cellulose coating, suggesting suitable cell compatibility of both uncoated and cellulose nanowhiskers coated scaffolds.

In previous studies, the typical cell morphology (osteoblastic phenotype with elongated polygonal structures and filopodias) of MG-63 cells has also been observed on 2D 45S5 BG-based pellets and 3D 45S5 BG-based scaffolds, ${ }^{42}$ including also on scaffolds with hybrid graphene-containing coating. ${ }^{55}$ The efficient cell infiltration into the 3D scaffolds and the cell proliferation in the internal region of the scaffolds are attributed to the typical large pore size and interconnected character of the pore structure prepared by foam replication method, which enable sufficient oxygen and nutrient diffusion. ${ }^{42}$ In the present study, the interconnected pore structure of scaffolds is well maintained and there is no significant change in the pore size after cellulose nanowhisker coating. In addition, the surface of cellulose nanowhiskers coated 45S5 BG scaffolds was quite hydrophilic.

In previous studies, cellulose nanowhiskers or their composites have been reported to be generally non-toxic to endothelial cells, ligament cells, fibroblasts and human adult adipose derived mesenchymal stem cells. ${ }^{33-40}$ The present study extends the understating of the cytocompatibility of cellulose nanowhiskers containing composite materials to osteoblast-like cells, and confirms the cytocompatibility of cellulose nanowhiskers coated 45S5 BG-based scaffolds to MG-63 cells. The present results also indicate that cellulose nanowhiskers coated 45S5 BG-based scaffolds may have potential for bone tissue engineering applications, although more thorough testing with relevant human cell lines such as mesenchymal stem cells, as well as in vivo testing, are required to confirm this potential.

\section{Conclusions}

45S5 BG-based scaffolds obtained by foam replication method were coated with cellulose nanowhiskers by dip coating method. The application of a cellulose coating improved the compressive strength and mechanical stability of the scaffolds, and the acellular in vitro bioactivity was maintained in the cellulose nanowhiskers coated scaffolds. In addition, in vitro experiments showed that the composite scaffolds were biocompatible for MG-63 cells. The results indicate that the obtained bioactive and biocompatible composite scaffolds are promising for bone tissue engineering. Further studies are required to investigate the degradation behavior of the scaffolds and the biocompatibility toward relevant cells for the intended applications, especially mesenchymal stem cells. 


\section{Acknowledgements}

Wei Li and Yaping Ding would like to acknowledge the China Scholarship Council (CSC) for financial support.

\section{References}

1 A. R. Amini, C. T. Laurencin and S. P. Nukavarapu, Crit. Rev. Bioeng., 2012, 40, 363-408.

2 V. Mourino and A. R. Boccaccini, J. R. Soc., Interface, 2010, 7, 209-227.

3 A. J. Salinas and M. Vallet-Regi, RSC Adv., 2013, 3, 1111611131.

4 L. L. Hench, J. Mater. Sci.: Mater. Med., 2006, 17, 967-978.

5 J. R. Jones, Acta Biomater., 2013, 9, 4457-4486.

6 A. A. Gorustovich, J. A. Roether and A. R. Boccaccini, Tissue Eng., Part B, 2010, 16, 199-207.

7 L.-C. Gerhardt and A. R. Boccaccini, Materials, 2010, 3, 38673910 .

8 S. Bose, M. Roy and A. Bandyopadhyay, Trends Biotechnol., 2012, 30, 546-554.

9 V. Mouriño, J. P. Cattalini, J. A. Roether, P. Dubey, I. Roy and A. R. Boccaccini, Expert Opin. Drug Delivery, 2013, 10, 13531365.

10 Q. Z. Chen, I. D. Thompson and A. R. Boccaccini, Biomaterials, 2006, 27, 2414-2425.

11 Q. Z. Chen and A. R. Boccaccini, J. Biomed. Mater. Res., Part A, 2006, 77, 445-457.

12 W. Li, P. Nooeaid, J. A. Roether, D. W. Schubert and A. R. Boccaccini, J. Eur. Ceram. Soc., 2014, 34, 505-514.

13 A. L. Metze, A. Grimm, P. Nooeaid, J. A. Roether, J. Hum, P. J. Newby, D. W. Schubert and A. R. Boccaccini, Key Eng. Mater., 2013, 541, 31-39.

14 L. Bertolla, I. Dlouhý, A. Philippart and A. R. Boccaccini, Mater. Lett., 2014, 118, 204-207.

15 D. M. Yunos, O. Bretcanu and A. R. Boccaccini, J. Mater. Sci., 2008, 43, 4433-4442.

16 B. Olalde, N. Garmendia, V. Sáez-Martínez, N. Argarate, P. Nooeaid, F. Morin and A. R. Boccaccini, Mater. Sci. Eng., C, 2013, 33, 3760-3767.

17 Q. Yao, P. Nooeaid, J. A. Roether, Y. Dong, Q. Zhang and A. R. Boccaccini, Ceram. Int., 2013, 39, 7517-7522.

18 C. Wu, Y. Ramaswamy, P. Boughton and H. Zreiqat, Acta Biomater., 2008, 4, 343-353.

19 D. Bellucci, A. Sola, P. Gentile, G. Ciardelli and V. Cannillo, J. Biomed. Mater. Res., Part A, 2012, 100, 3259-3266.

20 D. O. Costa, P. D. H. Prowse, T. Chrones, S. M. Sims, D. W. Hamilton, A. S. Rizkalla and S. J. Dixon, Biomaterials, 2013, 34, 7215-7226.

21 E. Palin, H. Liu and T. J. Webster, Nanotechnology, 2005, 16, 1828-1835.

22 J. Mitra, G. Tripathi, A. Sharma and B. Basu, RSC Adv., 2013, 3, 11073-11094.

23 D. C. Meng, M. Erol and A. R. Boccaccini, Adv. Eng. Mater., 2010, 12, B467-B487.
24 S.-I. Roohani-Esfahani, S. Nouri-Khorasani, Z. Lu, R. Appleyard and H. Zreiqat, Biomaterials, 2010, 31, 54985509.

25 L. Nie, D. Chen, Q. Yang, P. Zou, S. Feng, H. Hu and J. Suo, Mater. Lett., 2013, 92, 25-28.

26 D. Klemm, F. Kramer, S. Moritz, T. Lindström, M. Ankerfors, D. Gray and A. Dorris, Angew. Chem., Int. Ed., 2011, 50, 54385466.

27 S. Kalia, S. Boufi, A. Celli and S. Kango, Colloid Polym. Sci., 2014, 292, 5-31.

28 Q. Chen, U. P. de Larraya, N. Garmendia, M. LasherasZubiate, L. Cordero-Arias, S. Virtanen and A. R. Boccaccini, Colloids Surf., B, 2014, 118, 41-48.

29 R. M. A. Domingues, M. E. Gomes and R. L. Reis, Biomacromolecules, 2014, 15, 2327-2346.

30 S. Virtanen, J. Vartianen, H. Setala, T. Tammelin and S. Vuoti, RSC Adv., 2014, 4, 11343-11350.

31 K. Hua, D. O. Carlsson, E. Alander, T. Lindstrom, M. Stromme, A. Mihranyan and N. Ferraz, RSC Adv., 2014, 4, 2892-2903.

32 J. M. Dugan, J. E. Gough and S. J. Eichhorn, Nanomedicine, 2013, 8, 287-298.

33 R. Maren, D. Shuping, H. Anjali and L. Yong Woo, in Polysaccharide Materials: Performance by Design, American Chemical Society, 2009, vol. 1017, pp. 81-91.

34 A. P. Mathew, K. Oksman, D. Pierron and M.-F. Harmand, Carbohydr. Polym., 2012, 87, 2291-2298.

35 L. Alexandrescu, K. Syverud, A. Gatti and G. ChingaCarrasco, Cellulose, 2013, 20, 1765-1775.

36 A. P. Mathew, K. Oksman, D. Pierron and M.-F. Harmand, Macromol. Biosci., 2013, 13, 289-298.

37 L. Rueda, A. Saralegi, B. Fernández-d'Arlas, Q. Zhou, A. Alonso-Varona, L. A. Berglund, I. Mondragon, M. A. Corcuera and A. Eceiza, Cellulose, 2013, 20, 1819-1828.

38 X. Yang, E. Bakaic, T. Hoare and E. D. Cranston, Biomacromolecules, 2013, 14, 4447-4455.

39 W. Li, R. Guo, Y. Lan, Y. Zhang, W. Xue and Y. Zhang, J. Biomed. Mater. Res., Part A, 2014, 102, 1131-1139.

40 C. Zhou, Q. Shi, W. Guo, L. Terrell, A. T. Qureshi, D. J. Hayes and Q. Wu, ACS Appl. Mater. Interfaces, 2013, 5, 3847-3854.

41 L. L. Hench, J. Am. Ceram. Soc., 1998, 81, 1705-1728.

42 Q. Z. Chen, A. Efthymiou, V. Salih and A. R. Boccaccinil, J. Biomed. Mater. Res., Part A, 2008, 84, 1049-1060.

43 A. O'Sullivan, Cellulose, 1997, 4, 173-207.

44 T. Kokubo and H. Takadama, Biomaterials, 2006, 27, 29072915.

45 V. Karageorgiou and D. Kaplan, Biomaterials, 2005, 26, 54745491.

46 Q. Yao, P. Nooeaid, R. Detsch, J. A. Roether, Y. Dong, O.-M. Goudouri, D. W. Schubert and A. R. Boccaccini, J. Biomed. Mater. Res., Part A, 2014, 102(12), 4510-4518.

47 L. Francis, D. C. Meng, J. C. Knowles, I. Roy and A. R. Boccaccini, Acta Biomater., 2010, 6, 2773-2786.

48 W. Li, Y. Ding, R. Rai, J. A. Roether, D. W. Schubert and A. R. Boccaccini, Mater. Sci. Eng., C, 2014, 41, 320-328. 
49 M. Peroglio, L. Gremillard, J. Chevalier, L. Chazeau, C. Gauthier and T. Hamaide, J. Eur. Ceram. Soc., 2007, 27, 2679-2685.

50 M. Dressler, F. Dombrowski, U. Simon, J. Börnstein, V. D. Hodoroaba, M. Feigl, S. Grunow, R. Gildenhaar and M. Neumann, J. Eur. Ceram. Soc., 2011, 31, 523-529.

51 M. M. Erol, V. Mourino, P. Newby, X. Chatzistavrou, J. A. Roether, L. Hupa and A. R. Boccaccini, Acta Biomater, 2012, 8, 792-801.
52 W. Huang, Y. J. Wang, L. Ren, C. Du and X. T. Shi, Mater. Sci. Eng., C, 2009, 29, 2221-2225.

53 O. P. Filho, G. P. La Torre and L. L. Hench, J. Biomed. Mater. Res., 1996, 30, 509-514.

54 Y. Zhu and S. Kaskel, Microporous Mesoporous Mater., 2009, 118, 176-182.

55 P. Fabbri, L. Valentini, J. Hum, R. Detsch and A. R. Boccaccini, Mater. Sci. Eng., C, 2013, 33, 3592-3600. 